\title{
Impacto de la inmigración sobre el crecimiento de la renta per cápita de Ecuador (2001-2015)
}

\section{Impact of the immigration on the growth of the per capita income of Ecuador (2001-2015)}

Eduardo Germán Zurita

María Gabriela González

Eduardo Ramiro Dávalos

Universidad Nacional de Chimborazo, Ecuador

Autor para correspondencia: ezurita@unach.edu.ec,mggonzalez@unach.edu.ec, edavalos@unach.edu.ec

Fecha de recepción: 02 de Agosto de 2017 - Fecha de aceptación: 30 de Septiembre de 2017

\section{Resumen}

El análisis de los efectos de la inmigración sobre la renta per cápita tiende acrecentar el porcentaje de población en edad de trabajar integrándolos plenamente dentro del sistema productivo, que fomenta el crecimiento económico y el aumento del bienestar en la población. El trabajo busca determinar el impacto de la inmigración sobre el crecimiento de la renta per cápita de Ecuador a través del modelo de regresión lineal múltiple. Este modelo se basa en la teoría de la transición demográfica, con la premisa de que los inmigrantes se ven atraídos por las diferencias salariales de su país con respecto a otro. En conclusión, se obtiene una relación directa entre las variables independientes inmigración, tasa de empleo, productividad y la variable dependiente renta per cápita.

Palabras clave: empleo; inmigración; renta per cápita; productividad

\begin{abstract}
The analysis of the effects of immigration on per capita income tends to increase the percentage of the working age population by fully integrating them into the productive system, which fosters economic growth and increases well-being in the population. The paper seeks to determine the Impact of Immigration on the growth of per capita income in Ecuador through the multiple linear regression model. This model is based on the theory of demographic transition, with the premise that immigrants are attracted by the wage differences of their country with respect to another. In conclusion we obtain a direct relation between the independent variables immigration, employment rate, productivity and the dependent variable per capita income.
\end{abstract}

Key words: employment; immigration; income per capita; productivity 


\section{Introducción}

A lo largo de la historia, el factor demográfico ha desempeñado un papel fundamental en las economías del mundo, constituyéndose en indispensable para la formación de la fuerza laboral y el capital humano. Conforme crece la inversión, la población inmigrante va acumulando conocimiento y asimilación a nuevos equipos tecnológicos lo que contribuye significativamente en el acrecentamiento de la productividad y el bienestar de la población en los países receptores como también en los países de origen.

Sin embargo, las evidencias empíricas señalan que no todos los inmigrantes tienden a aportar efectivamente en el incremento de la renta per cápita, ya que otros influyen en las personas con actividades ilícitas perjudiciales para la población.

En efecto, el objetivo principal del presente trabajo es determinar el impacto de la inmigración en el crecimiento de la renta per cápita, desde el 2001; año en el cual, la economía ecuatoriana empezó a equilibrarse, tras superar la peor crisis financiera del siglo, esto debido al nuevo sistema monetario adoptado en el país. Por otro lado, el crecimiento de la renta per cápita constituye uno de los fines de la política económica, a través del incremento de la fuerza del trabajo y el capital humano que fomenta la infraestructura productiva y el empleo.

La investigación se desarrolló dentro del modelo neoclásico de la transición demográfica, la cual propone que el crecimiento de la población de inmigrantes estimula positivamente la actividad económica y el bienestar de los habitantes.

\section{Planteamiento Teórico}

Patiño (2014), afirma que: "La inmigración es el movimiento de un ser humano o de un grupo de seres humanos que van de un espacio sobre la tierra a otro, de un punto A hacia un punto B" (p. 5).

Para (Oyarzun, 2008) la inmigración como tal, obedece a varias causas entre las cuales probablemente el aspecto económico sea el principal motivo para inmigrar, ya que muchas personas buscan en otros países mayores ingresos o un mejor nivel de vida, pero también existen otras causas sociales que les obligan a abandonar su país y buscar mejores días en otro lugar.

Los efectos que más se estudian en relación a los efectos económicos que pueden provocar la inmigración son: los cambios en la oferta de trabajo, cambios en la productividad, cualificación, empleo, niveles de salarios y la distribución de la renta.

\section{Crecimiento de la Renta per cápita} (p. 56)

Según (Dornbusch, 2008) "la renta per cápita es el cociente entre el PIB y la población”.

Este es un indicador que sirve para comparar el nivel de bienestar que tienen distintos países. Sin embargo, un país con mayor crecimiento del PIB y con índices poblacionales bajos, 
tendrá mayor nivel de bienestar, especialmente si la distribución de la renta no es desigual. Cuando el crecimiento poblacional es alto, la relación entre indicadores de salud, educación y satisfacción de necesidades es menor, ya que no necesariamente un país con elevado crecimiento del PIB goza de mayor bienestar, porque depende del crecimiento demográfico.

La renta per cápita es un indicador del crecimiento económico por cada habitante y se lo calcula de la siguiente manera.

Renta per cápita $=\mathrm{PIB} / \mathrm{N}^{\circ}$ de habitantes

\section{Inmigración y Renta per cápita}

En lo que se refiere a la inmigración y la renta per cápita Oglietti (2006) afirma:

La ley de los rendimientos decrecientes de Malthus predomina hasta nuestros tiempos cuando se analiza el impacto demográfico en el crecimiento económico de un país. En teoría persiste que como consecuencia de los rendimientos decrecientes el crecimiento de la población reduce la productividad. Pero esta idea está siendo estudiada ya que hay muchos quienes entienden que el crecimiento de la población puede tener un impacto positivo sobre el crecimiento de la renta per cápita. (p.89)

Así (Avendaño, 2007) considera que los cambios en la población son importantes antes que las cifras agregadas y en lo que respecta al impacto demográfico el análisis de las transiciones es mucho más esclarecedor que el de los estados estacionarios. La población inmigrante jugaría un papel muy importante ya que aporta individuos que pueden formar parte de la fuerza laboral lo que estimularía la economía.

Los procesos migratorios se dan por exceso de oferta de mano de obra en los países de origen y a su vez por un exceso de demanda en el país receptor, sin embargo, no todos deciden inmigrar. Principalmente lo hacen personas que tienen formación, personas con trabajos mal remunerados o que no cuentan con trabajo que se ven impulsados a migrar en busca de mejores condiciones de vida.

Este autor afirma que cuando una economía experimenta un crecimiento necesita de un período mínimo de 16 años para que los individuos nacionales ingresen al mercado laboral, mientras que la llegada de inmigrantes proporciona esa fuerza laboral sin tener costos de formación. A lo expuesto es necesario mencionar que los inmigrantes suelen ser personas con deseo de realización que buscan mejorar sus condiciones.

Existe un vínculo entre la inmigración y la renta per cápita, debido a que la población inmigrante juega un papel significativo, ya que el aporte de los mismos puede formar y ser parte de la fuerza laboral, por consiguiente, estimulará la economía. La inmigración tendrá un impacto positivo en la renta a través de porcentajes de la población en edad de trabajar y en la tasa de empleo de la economía, sin embargo, el proceso migratorio también puede tener un efecto en la productividad. 


\section{Metodología}

El modelo de la investigación se basa en la teoría de la "transición demográfica" de la escuela neoclásica; con la premisa de que los inmigrantes se ven atraídos por las diferencias salariales de su país con respecto al otro; y que el incremento del factor migratorio estimula positivamente la actividad económica y, por tanto, el aumento del bienestar en la población y el crecimiento y desarrollo económico sostenible.

El planteamiento del modelo econométrico, intenta determinar el impacto de la inmigración sobre el crecimiento de la renta per cápita ecuatoriana, para el período: 2001-2015. La variable dependiente es la renta per cápita, con el indicio de que un país es rico, si sus habitantes producen más. Por otra parte, las variables independientes o explicativas fueron, el componente demográfico de inmigración, es decir, la cantidad de inmigrantes que ingresaron al país; la tasa de empleo; y la productividad.

La estimación del modelo econométrico, se desarrolló a través del modelo clásico de regresión lineal múltiple, para el período 2001 - 2015; con datos semestrales; para un total de 30 observaciones, transformados en valores logarítmicos. A partir de la siguiente ecuación:

$$
\mathrm{Y} \_\mathrm{i}=\beta_{-}^{\wedge} 0+\beta_{-}^{\wedge} 1 \mathrm{X} \_1+\beta_{-}^{\wedge} 2 \mathrm{X} \_2+\beta_{-}^{\wedge} 3 \mathrm{X} \_3+\mathrm{e} \_\mathrm{i}
$$

Yi: Renta per cápita $=$ PIB/Población

X1: Factor demográfico de inmigración (números de personas que han ingresado al país)

X2: Tasa de empleo=Población ocupada/población en edad de trabajar (se tomó los datos de la población ocupada o con empleo registrados en el INEC)

X3: Productividad= PIB real/ población ocupada

ei: Perturbación estocástica

Al realizar el proceso de función logarítmica se obtuvo:

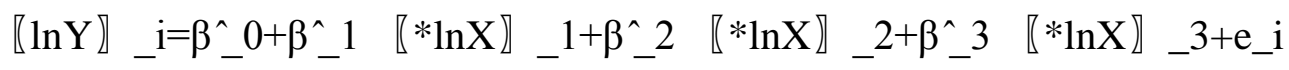

$\beta \_0=$ Intercepto del modelo

$\beta \_\mathrm{i}=$ Elasticidad de los coeficientes

e_i = Término de perturbación estocástico

\section{Resultados}

\section{Evolución de la Inmigración}

Históricamente, el Ecuador ha sido considerado un país emisor de migrantes y, no ha sido un país con importante tradición inmigratoria; sin embargo, el incremento de la movilidad de la población en general también significó un crecimiento en la llegada de población extranjera al país. Según, los datos del INEC, durante la década de los noventa, la inmigración se registró en alrededor de 65.147 inmigrantes, provenientes de distintos países latinoamericanos quienes llegaron afectados de las guerras civiles y de los fenómenos naturales, así como también afectados de las crisis económicas, sociales y políticas en sus países de origen. 
Según, el (Instituto Nacional de Estadística y Censos, 2010) del total de extranjeros residentes en el Ecuador, el 34.2\% son oriundos de la vecina república de Colombia. El segundo grupo de inmigrantes en orden de importancia provendría de los Estados Unidos de Norteamérica y el tercero y cuarto lugar lo ocuparían los nacidos en Perú y Chile $(3.8 \%$ y $3.1 \%$, respectivamente).

El gráfico 1, muestra la evolución de la población demográfica de inmigración, a partir del año 2001, en el cual, se contabilizó alrededor 640 mil personas que ingresaron al país, por motivos turísticos, laborales, académicos y servicios profesionales; al mismo tiempo; varios de los extranjeros llegaron atraídos por los montos salariales, especialmente, los inmigrantes de los vecinos países (peruano, colombianos y venezolano) que son beneficiados de la moneda ecuatoriana y los ingresos altos al convertir el dólar en la moneda de sus países, por medio de las actividades licitas o ilícitas, entre otros.

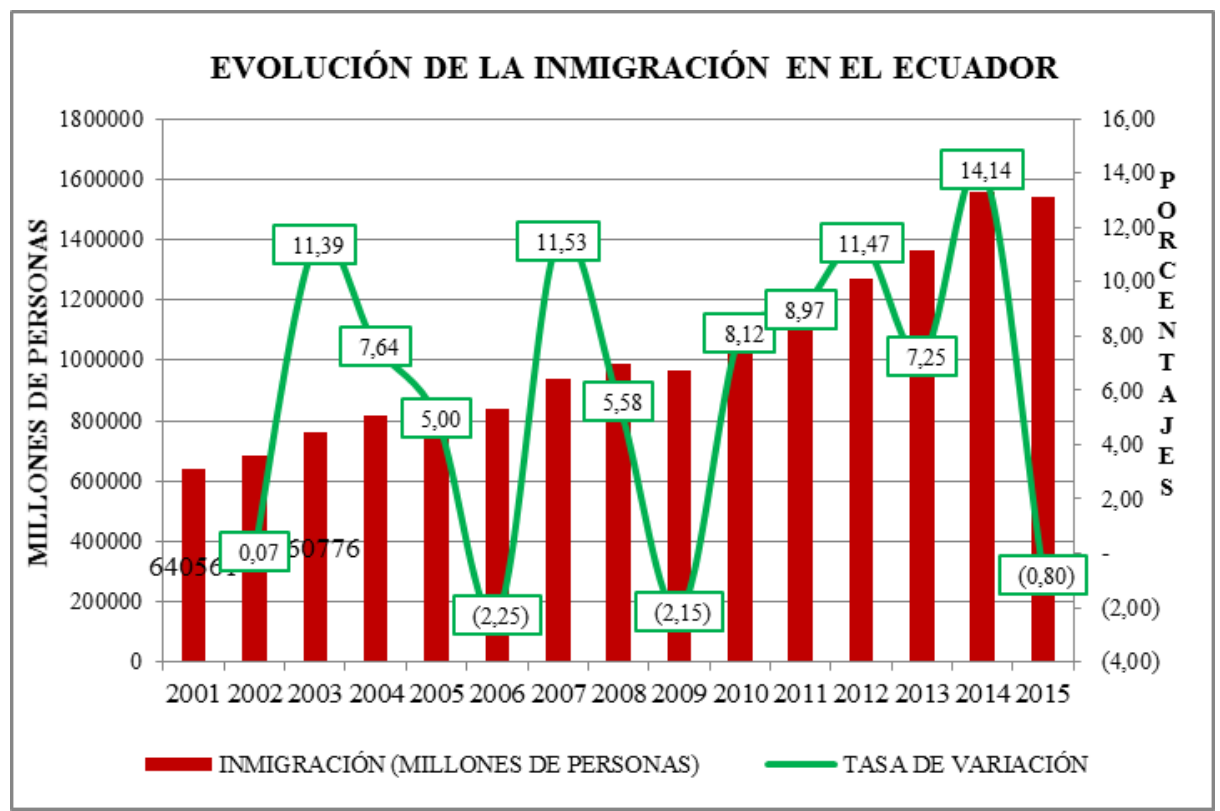

Gráfico 1 Evolución de la Inmigración en el Ecuador Período: 2001-2015 (En millones \& porcentajes)

Fuente: Instituto Nacional de Estadística y Censos Elaborado por: Autores

Como era de esperarse, los inmigrantes siguieron ascendiendo año tras año, siendo así, en el año 2012, se contabilizó alrededor 1.271 mil inmigrantes, experimentando una tasa de variación del 19\% al 2013 en promedio, mientras que en los dos últimos años de análisis, la inmigración experimentó un descenso importante hasta un promedio del 13\% de tasa de variación, debido a la caída de los precios internacionales de petróleo, consecuentemente se disminuyó plazas de trabajo y caída de la inversión pública, privada y las inversiones extranjeras en el país.

\section{Factor demográfico en el país}


El factor demográfico ecuatoriano, se desprende de la desintegración de la población en edad en trabajar sobre la población total; la población en edad de trabajar durante los años 1990 y 2005 creció en un $2.9 \%$ promedio anual superior a la tasa de crecimiento de la población total que era de $1.69 \%$, este crecimiento fue debido a la migración desde el campo hacia la ciudad en búsqueda de plazas empleo y con la idea de mejorar la condición económica de la familia, ofreciendo mejores niveles de estudios a los hijos. Por otra parte, la población joven en el 2005 representaba el 47,1\% de la población en edad de trabajar, no todo este grupo de población participaba en la fuerza laboral, debido a los avances en la escolarización de los jóvenes; la cual, reflejaba en la participación de la población más joven en la Población Económicamente Activa.

En primera instancia, ha existido un crecimiento acentuando de la Población en Edad de Trabajar o personas más de 15 y 64 años en comparación a las personas menores de 15 años y de aquellas mayores de 65 años. En este contexto, para el último período de análisis, la población del Ecuador se divide en: $31 \%$ menores de 15 años, $62 \%$ personas entre el 15 y 64 años de edad y $7 \%$ mayores de 65 años. Esto refleja que en el país, se mantiene en un proceso constante de aumento proporcional de la población en edad de actividad laboral, proceso donde se espera una expansión económica importante derivada de alto stock de población en edad productiva en comparación a la población dependiente, según (CEPAL, 2014)

Además, los estudios del (CEPAL, 2014) muestra que la PET, clasificada cómo la población de 15 y más, hay un incremento en el porcentaje de la población entre 2007 y 2014 que se explica por el aumento de estudiantes y de personas que por sus capacidades físicas o mentales no pueden trabajar, la restante proporción de la PET se considera población económicamente activa (PEA) y son aquellas personas que están trabajando o desean hacerlo. De los cuales, la PEA, entre 2007 y 2015 el 95\% se encuentra trabajando en relación de dependencia o se genera su propio empleo. La ocupación de este sector se concentra principalmente en la agricultura, los servicios, manufactura, etc., acumulando a más de 60\% de empleo en 2015.

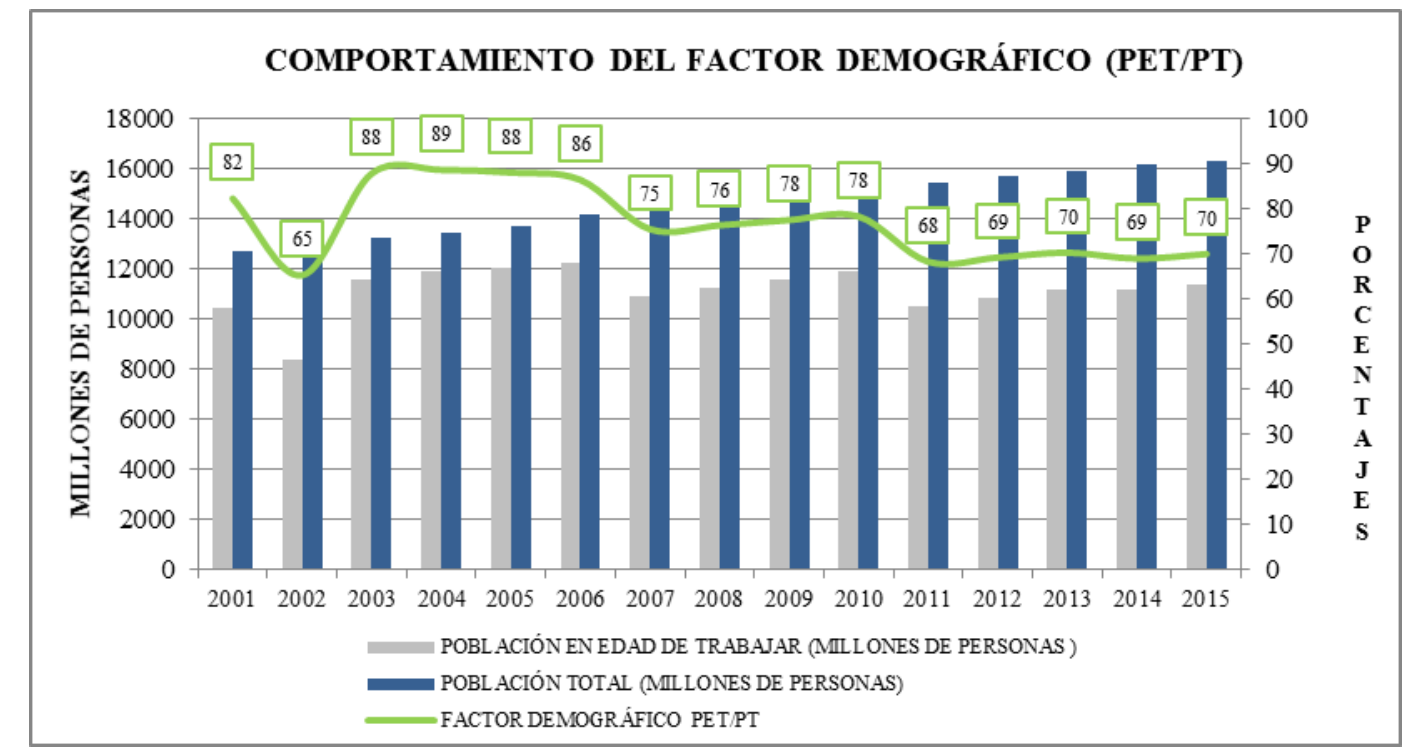

Gráfico 2 Comportamiento del factor demográfico - Período: 2001-2015 (Millones \& porcentajes) Fuente: Instituto Nacional de Estadística y Censos - Elaborado por: Autores 
En el gráfico 2, muestra el comportamiento del factor demográfico a partir del año 2001; en el cual, se presenta una cantidad de 10 millones de personas en edad de trabajar, pero hasta el 2002 la población en edad de trabajar disminuyó en un -20\% debido a un alto índice de emigración debido a la crisis financiera que se dio en el país. Al 2003, la población creció representando un $88 \%$ del factor demográfico, desempeñando significativamente en la actividad laboral del país. A partir de año 2007 al 2010 la población en edad de trabajar sigue creciendo ya que la población total también crece del 2001 al 2015 la población creció a 16 millones de habitantes siendo 11,399 habitantes que están en edad de trabajar representando alrededor de $70 \%$ del factor demográfico.

\section{Situación laboral en el país}

En los años 90, en el marco de la reducción del estado y la búsqueda de mayor flexibilización cambio la estructura del mercado ocupacional. La precarización laboral se profundizó con la tercerización e intermediación laboral, eliminada posteriormente con el mandato 8 de la Asamblea Constituyente en mayo del 2008.

Según datos del INEC, las personas ocupadas de 1992 al 1998 fluctúo entre 32\% al 39\%, en tanto en 1999 se ubicó en $27 \%$, el porcentaje más bajo de todo el período. Ecuador se ha caracterizado por sus deficientes resultados, el cual, se manifiesta a través de la alta y creciente proporción de empleos informales y al escaso dinamismo del sector moderno en la generación de empleo. Se dio así un aumento de participación en el sector informal sobre la población ocupada, lo cual ascendió de $43 \%$ al $51 \%$ entre 1998 y el año 2000.

En la década de los 2000 el crecimiento económico del país presento una importante recuperación, pero este crecimiento fluctuante no aseguró condiciones de estabilidad para la inversión y no permitió reducir las elevadas tasas de desempleo e informalidad. Sin embargo, con la dolarización, las reformas en el mercado laboral tuvieron también tuvieron efectos negativos tanto sobre los salarios reales, en el porcentaje de la población que se encuentra adecuadamente empleada, estos riesgos se dieron en términos del incremento en la vulnerabilidad estable del empleo asalariado.

Según, la encuesta nacional de empleo la población ocupada eran de alrededor del 30\% del total de la Población económicamente activa urbana del año 2000, en los años 2002 al 2004 constituyen el $37 \%$ y en el 2005 son el $36 \%$ el total en donde se registra una diferencia al considerarlo por género. A partir del año 2005 mientras la población en edad de trabajar ofertaba su trabajo en un $62 \%$ activamente, el $57 \%$ de esta población se encontraba en algún tipo de ocupación. 


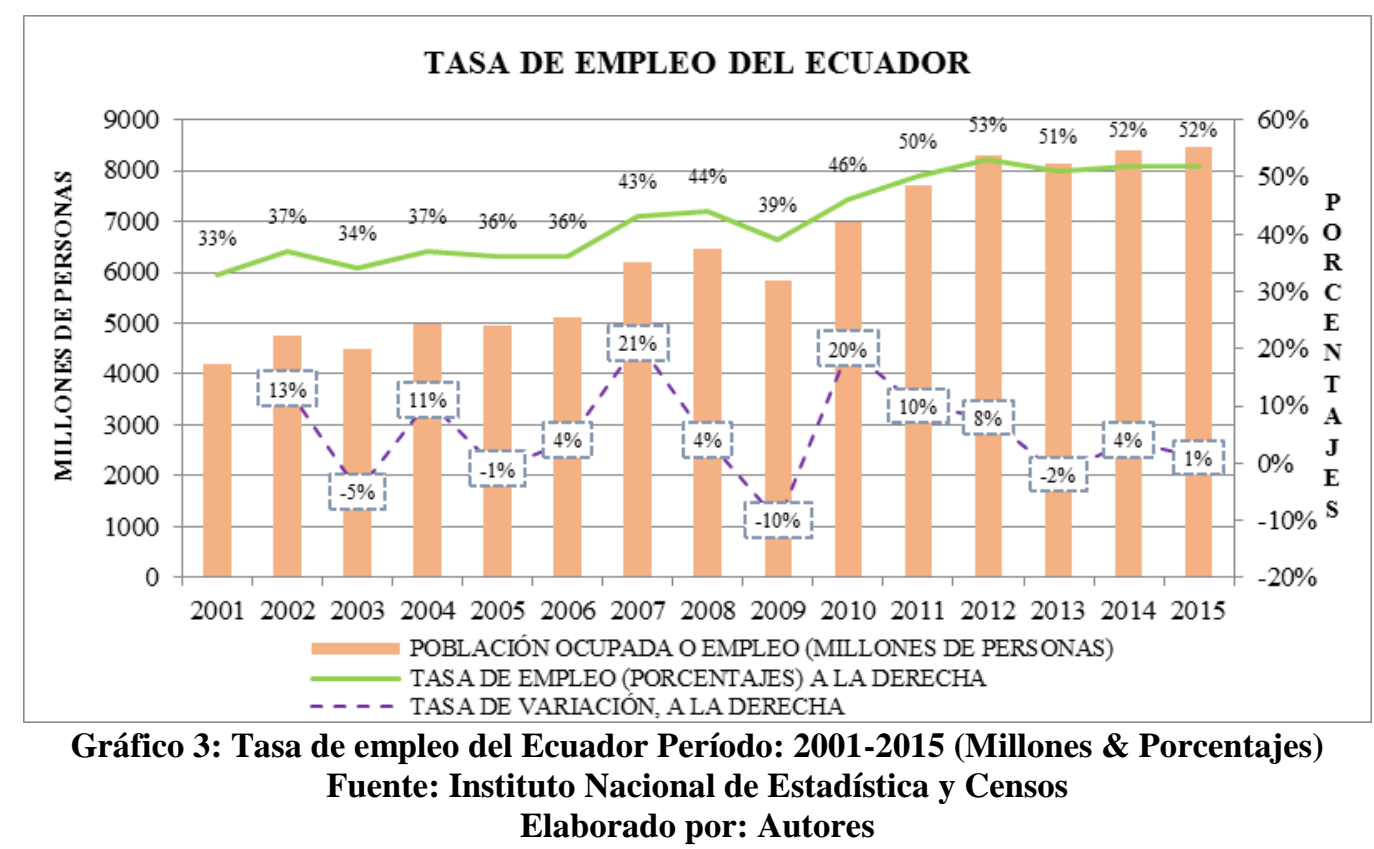

En el grafico 3, muestra la evolución de la tasa de empleo en el Ecuador, el mismo que se situó en un 55\% del total de un 4.198 millones de personas ocupadas, esta cifra se debe al número elevado de emigración que se dio en el país, mientras que para el año siguiente, la población ocupada fue de 4.746 millones de personas, representando el 13\% de variación; la tasa de empleo para el 2006 se sitúo en un 36\% del total de la población ocupada, en cambio al 2007 la población ocupada creció a 6 millones a personas de un total de 14 millones de habitantes a nivel nacional.

Mientras que desde al año 2009 a 2012, la población ocupada creció de 5 a 8 millones de personas ocupadas, llegando a situarse la tasa de empleo de $39 \%$ a un $51 \%$ respectivamente, así mismo, en los últimos tres años, la población en edad de trabajar se mantuvo en 8 millones de personas, variando a la tasa de empleo de $51 \%$ al $52 \%$ del total de la población ocupada.

\section{Comportamiento de la Productividad}

En los sectores transables que comprende la suma del valor agregado bruto de la agricultura, pesca, caza, silvicultura y otras minas e industrias manufactureras, se reflejó una productividad del trabajo decreciente; cabe mencionar que en el año 2001 aún se conservaba los rezagos de la crisis económica generada en el 99, por lo que el PIB no se había recuperado lo suficiente respecto a los años previos a la misma, esto se evidenció principalmente en el sector de las finanzas y comercio.

Los niveles de productividad muestran un crecimiento sostenido desde el inicio del milenio, es decir, la productividad en el período entre 2000 y 2006 creció a un ritmo de $2.28 \%$ anual, mientras que en los siguientes siete años (2014), la productividad creció en promedio $3.5 \%$ anual. Durante los mismos períodos, la productividad de la industria manufacturera aumentó en $1.07 \%$ anual y $3.4 \%$ anual, respectivamente. Así mismo, de otros sectores de actividad económica como el sector agropecuario, servicios, etc. 
El Ecuador posee una estructura productiva (productividad) enfocada principalmente en actividades primarias y servicios. Entre las actividades primarias destaca la extracción de minas y petróleo que entre 2007 y 2015 participó con el 10 \% en el PIB (sin considerar los otros elementos del PIB) del país, a la vez que es la principal fuente de divisas por concepto de exportaciones. La industria manufacturera en el país, es un sector con mayor encadenamiento y que concentra la mayoría del comercio nacional, que concentra el $13.3 \%$ del PIB en el último período de análisis. Según el (CEPAL, 2015)

En el grafico $\mathrm{n}^{\circ} 4$ muestra el comportamiento de la productividad del Ecuador durante el período de análisis, siendo uno de los determinantes que influyen en el crecimiento de la renta per cápita. En el cual, a partir del año 2001, la productividad fue de 9.3 obteniendo una variación de siete puntos porcentuales al año 2002, en cambio al llegar al 2006, la productividad fue de 9.8 con una población ocupada de 5 millones de habitantes; en los dos años posteriores, del (2008 a 2009) la productividad laboral subió de 8.4 a 9.4 respectivamente, teniendo una tasa de variación de $11 \%$.

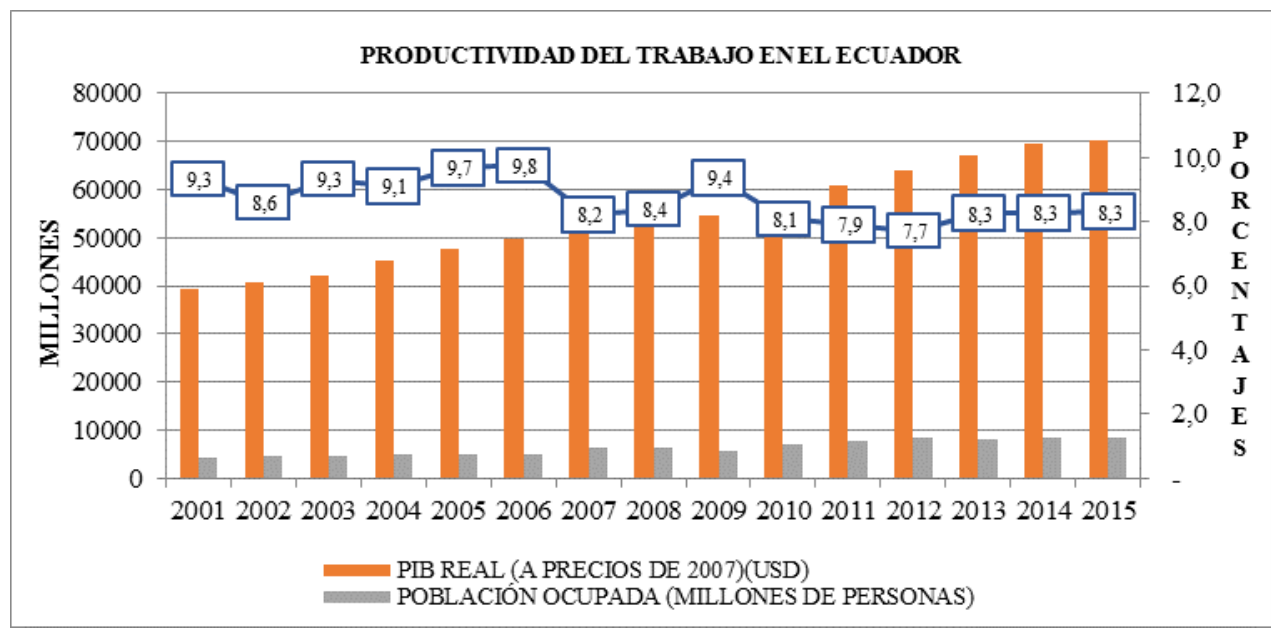

Gráfico 4: Comportamiento de la Productividad en el Ecuador Período: 2001-2015 (Precios \& millones) Fuente: BCE; Instituto Nacional de Estadística y Censos Elaborado por: Autores

Consecuentemente, a partir del 2010, la productividad de país, se evidenció un descenso con una población ocupada de 6 a 8 millones de personas ocupadas con una tasa de variación de -4.64\%; mientras que, en los últimos años de análisis, la productividad se mantiene en 8.3 con una tasa de variación del 63 punto porcentual debido a crisis económica que se vive en el país, a consecuencia de la caída de los precios del petróleo y por ende la reducción de la inversión pública y privada.

\section{Evolución de la renta per cápita en el Ecuador}

La renta per cápita representa el valor de los bienes y servicios finales durante un año dado, el cual le correspondería a cada habitante. El crecimiento del PIB en el Ecuador en el período pre dolarización fue menor que el período de la economía dolarizada. Entre 1990-1999 
el crecimiento del producto interno bruto fue de un promedio de $1.8 \%$ mientras que en la década siguiente fue de $4.4 \%$ con un significativo aumento.

Durante la década de los 90, se caracterizó por varios momentos de inestabilidad económica provocada por la crisis financiera, provocando la quiebra de las empresas locales, especialmente, las pequeñas y medianas empresas que funcionaban con un capital reducido, así produjo la destrucción de empleo, provocando la perdidas de salario y por tanto, la pérdida del poder adquisitivo de las familias y el empeoramiento de las condiciones de trabajo y con ella la migración de las personas, perdiendo así, un $20 \%$ de la población económicamente activa, por lo que el PIB del Ecuador decreció en un -7.3\%, según los indicadores del Banco Central del Ecuador. Frente a estos acontecimientos suscitados en el país, el presidente de Ecuador, en curso decidió implementar el sistema de la dolarización a partir del año 2000, con el propósito de controlar la inflación y frenar la crisis económica de raíz.

Las cifras del Banco Central del Ecuador, muestran que, en la década anterior de la dolarización, se evidencio un estancamiento de la economía en el Ecuador con niveles del PIB per cápita promedios de $-0.2 \%$, cabe señalar que la caída del PIB per cápita se dio en el año 1999, período de la pre dolarización con $-7.6 \%$ de caída en el PIB per cápita. Durante la dolarización el crecimiento promedio de este indicador, en los años 2004 y 2008, registró tasas de crecimiento de $6.4 \%$ y $4.0 \%$ respectivamente. Es importante destacar, en los mismos años de la década de los 90, el PIB per cápita ascendió de USD 1.336 por persona, hay que recordar que reducir los niveles de pobreza, el país necesitaba tasas altas de crecimiento económico per cápita y sostenibles en el tiempo que lamentablemente esto no ha ocurrido.

En el año 2009, la tasa de ingresos per cápita cayó a -1.2\%, debido a la crisis económica internacional, la caída de remesas, reducción de los precios del petróleo y su demanda internacional. En los siguientes años, muestra un crecimiento de $6.1 \%$ en el 2011, gracias al dinamismo que se dio en el sector minero y petrolero, impulso a la inversión pública y el aporte de los sectores industriales, manufactureros y de servicios.

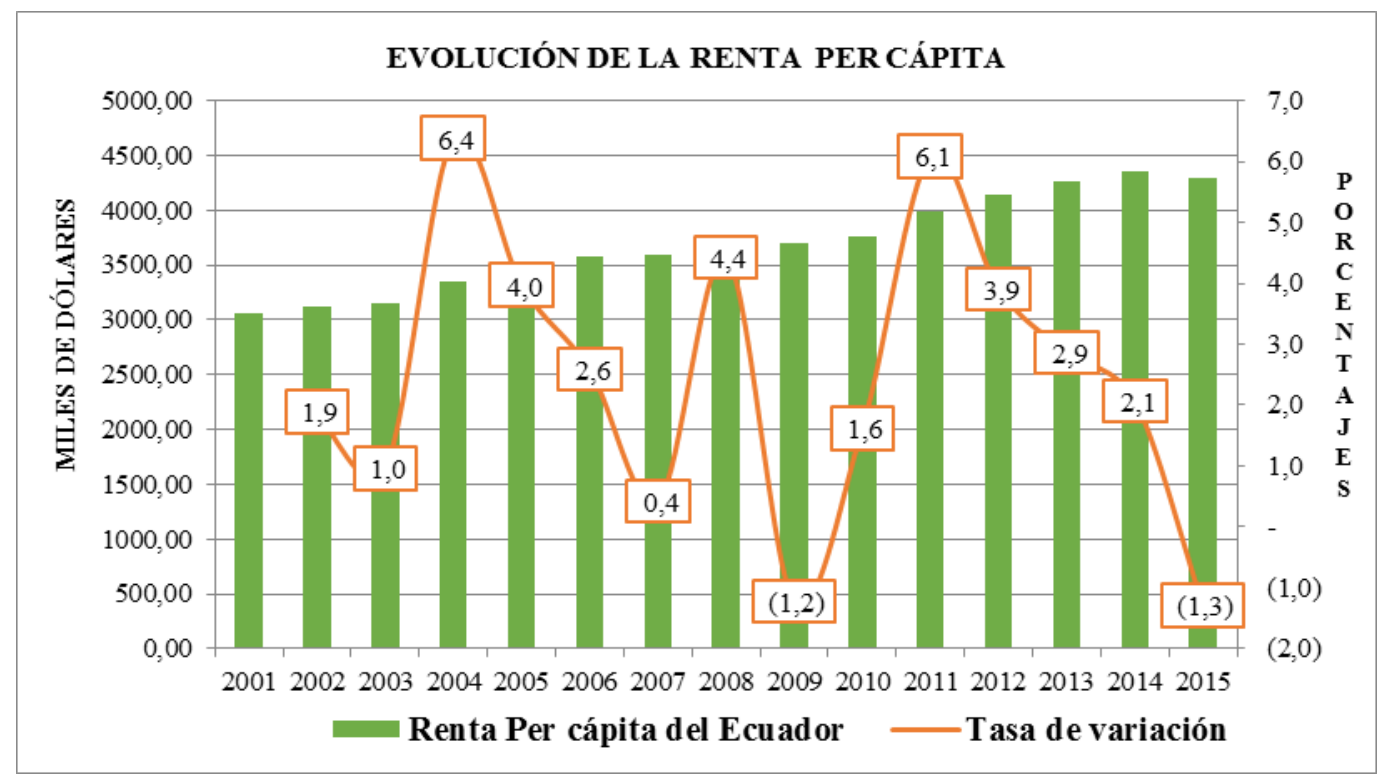

Gráfico 5: Evolución de la Renta per cápita Período: 2001 - 2015 (Dólares por persona \& Porcentaje) 


\section{Fuente: Banco Central del Ecuador Elaborado por: Autores}

Como se puede observar en el grafico $\mathrm{n}^{\circ} 5$, la evolución de la renta per cápita a precios reales constantes con año base 2007, presenta una tendencia creciente positiva, registrándose en USD 3.062.00 dólares al año en el año 2001, y en el 2005 USD 3.484.00 debido al incremento significativo del valor agregado petrolero con una tasa de variación del 4\%; sigue la tendencia creciente hasta el año 2008 que se registró en 3,748.00 dólares por persona; mientras que en el 2009 se reduce un punto porcentual, reduciéndose la renta per cápita, esto se dio debido a la crisis económica que empezó a enfrentar el país, por la reducción del precio del petróleo a nivel mundial y la disminución de la remesas.

Según los datos del Banco Central del Ecuador en el 2014 la renta se registró en USD 4.354.00 dólares por persona hasta 4.298 .00 en el año 2015 lo que representa $1.3 \%$, a causa de la reducción de la producción del petróleo y el precio a nivel internacional.

\section{Inmigración y Renta per cápita}

En Ecuador el fenómeno inmigratorio es más reciente, tener conocimiento de cuántos desean venir y de qué país provienen es una herramienta importante a la hora de tomar decisiones adecuadas en cuanto a destinación de recursos y políticas de integración. Como por ejemplo las políticas públicas que requieren de cierta anticipación en la información en cuanto a su extensión y localización, de aquí surge la importancia de estudio ya que la inmigración también juega parte en nuestra economía. Así, según los datos del INEC a partir del año 2001 a 2010 la inmigración creció a 1,047.098 millones de inmigrantes, atraídos por la moneda y por los puestos dejados por las migrantes ecuatorianas.

La (Revista Analítika, 2015), menciona que implícita o explícitamente se asume que las diferencias del PIB per cápita son la fuerza principal que motiva la migración laboral.

En el gráfico 6 se aprecia la relación entre el factor demográfico extranjero (inmigrantes) que llegaron al país y el crecimiento del Producto Interno Bruto per cápita en últimos 15 años; en el período las variables muestran una tendencia creciente, en ese sentido la renta per cápita tuvo un incremento significativo $40.37 \%$, con algunos desajustes y descensos en ciertos años 2006, 2009 y 2015, a consecuencia de la caída de los precios del petróleo y la crisis financiera mundial y otros factores. 


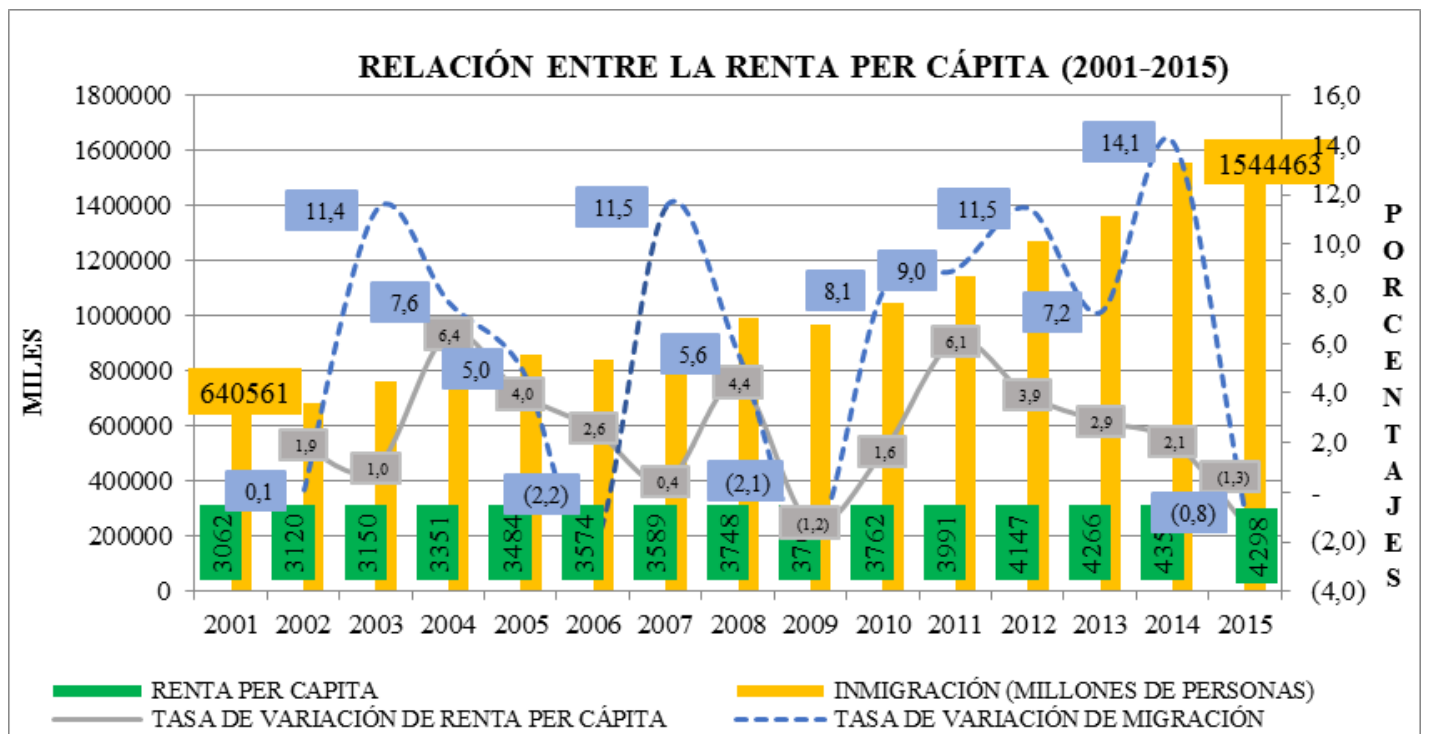

Gráfico 6: Relación entre Renta per cápita e Inmigración Período: 2001-2015 (Dólares por personas y millones)

Fuente: Banco Central del Ecuador; Instituto Nacional de Estadística y Censos Elaborado por: Autores

Por su parte, la inmigración al igual que la renta per cápita muestra un ascenso importante registrando para el año 2015 alrededor de 1.544.463 extranjeros en el país, de (640.561 inmigrantes contabilizados en 2001), experimentando una tasa de variación de $141 \%$. De los cuales, gran parte de la población extranjera se hallan dedicadas a las actividades económicas y comerciales como restaurantes, servicios de hotelería, distribución de suministros informáticos, servicios profesionales, especialmente en el área de salud y educación.

\section{Aplicación y Resultado del modelo Econométrico}

En la aplicación del modelo se realizó diversas pruebas econométricas de acuerdo a los lineamientos del método de mínimos cuadrados ordinarios (MCO), como son: linealidad, homocedasticidad, normalidad y de no auto correlación, esto con la finalidad de no recaer en la regresión espuria.

Tabla 1: Estimación del modelo econométrico

\begin{tabular}{|c|c|c|c|c|}
\hline & $\begin{array}{r}\text { Dependen } \\
\text { Method } \\
\text { Date: 11/2 } \\
\text { Sample } \\
\text { Included }\end{array}$ & $\begin{array}{l}\text { riable: LOG } \\
\text { ast Squares } \\
\text { Time: 06:3 } \\
\text { 1S1 2015S2 } \\
\text { ervations: } 30\end{array}$ & & \\
\hline Variable & Coefficient & Std. Error & t-Statistic & Prob. \\
\hline $\mathrm{C}$ & 1.585652 & 0.371186 & 4.271856 & 0.0002 \\
\hline LOGX & 0.218814 & 0.052705 & 4.151710 & 0.0003 \\
\hline LOGX1 & 0.493946 & 0.124276 & 3.974602 & 0.0005 \\
\hline LOGX2 & 0.490234 & 0.138259 & 3.545776 & 0.0015 \\
\hline R-squared & 0.970817 & \multicolumn{2}{|c|}{ Mean dependent var } & 7.518175 \\
\hline Adjusted R-squared & 0.967449 & \multicolumn{2}{|c|}{ S.D. dependent var } & 0.116489 \\
\hline
\end{tabular}




\begin{tabular}{cccc}
\hline S.E. of regression & 0.021017 & Akaike info criterion & -4.763428 \\
Sum squared resid & 0.011484 & Schwarz criterion & -4.576602 \\
Log likelihood & 75.45142 & Hannan-Quinn criter. & -4.703661 \\
F-statistic & 288.3051 & Durbin-Watson stat & 1.525701 \\
Prob(F-statistic) & 0.000000 & & \\
\hline
\end{tabular}

Elaborado por: Autores

Al observar los resultados finales de la estimación del modelo de la tabla 1 se encontró que los valores de los coeficientes corresponden a la teoría económica; es decir, muestran una relación directa entre las variables independientes (la inmigración, tasa de empleo y productividad) y la variable dependiente renta per cápita.

Expresando la siguiente ecuación:

$$
\text { LOGY }=1.58565+0.21881 * \text { LOGX1 + 0.49394*LOGX2 + 0.49023*LOGX3 }
$$

$\checkmark \quad \boldsymbol{\beta} \_=1.5856$, representa al valor constante o el intercepto del modelo, el cual significa que al permanecer constante (cero) los valores de las variables independientes, el modelo tendrá un valor constante de 1.58\%; sin embargo, no posee ninguna interpretación económica viable.

$\checkmark \quad \boldsymbol{\beta} \mathbf{1}=\mathbf{0 . 2 1 8 8}$, corresponde al coeficiente elasticidad parcial de factor demográfico de inmigración con respecto a la renta; la cual, significa que al mantener constante las variables independientes: tasa de empleo y productividad. Un incremento de $1 \%$ en el número de inmigrante en el país, estimula un aumento de $0.21 \%$ en la renta per cápita.

$\checkmark \quad \boldsymbol{\beta} 2=\mathbf{0 . 4 9 3 9}$, este valor representa a la elasticidad parcial de la tasa de empleo respecto a la renta la misma que indica que al mantener constante las variables independientes inmigración y productividad. Un incremento de $1 \%$ en la tasa de empleo, provoca un aumento de $0.49 \%$ en la renta per cápita

$\checkmark \quad \boldsymbol{\beta} \mathbf{3}=\mathbf{0 . 4 9 0 2}$, por último, el coeficiente parcial de la productividad con respecto a la renta indica que, al mantener constante, los valores de la inmigración y la tasa de empleo; un acrecentamiento de 1 un punto porcentual de la productividad, provoca un incremento de $0.49 \%$ en la renta cápita.

De acuerdo, a los resultados de tabla anterior, el coeficiente parcial de la variable tasa de empleo es más elevado y explica mejor el impacto sobre la variable dependiente renta per cápita que la inmigración y productividad.

Analizado en forma individual los resultados del modelo de regresión, se concluye que los coeficientes de las elasticidades parciales $\beta \mathcal{1}, \beta 2, \beta 3$; son estadísticamente significativos a un nivel de significancia $5 \%$. 
El coeficiente de determinación R2 = es elevado (0.970817), lo significa que alrededor de un $97 \%$ de bondad de ajuste, es decir, existe fuerte grado de relación entre la renta per cápita y las variables explicativas.

El coeficiente de R2 ajustado es 0.967449 , lo que indica que la variación en la variable dependiente que es explicado por la variable independiente considerando el número de variables y el tamaño de muestra alcanza un $96 \%$.

\section{Comprobación de Hipótesis}

Para determinar, el impacto de la inmigración sobre el crecimiento de la renta per cápita del Ecuador, período 2001-2015; se utilizó la prueba significancia t, a través del coeficiente de regresión parcial individual sobre el factor demográfico de inmigración.

Planteamiento de la hipótesis:

$\mathbf{H 0}=\boldsymbol{\beta 1}=\mathbf{0 :}$ La inmigración no impacta en el crecimiento de la renta per cápita.

H1 $=\boldsymbol{\beta} 1 \neq$ 0: La inmigración impacta en el crecimiento de la renta per cápita.

El valor de t-students es $=4.151710$, con 30 observaciones y 26 grados de libertad, a un $5 \%$ del nivel de significancia. El valor crítico de $\mathrm{t}$ de la tabla $=2.201$, a un $5 \%$ del nivel de significancia.

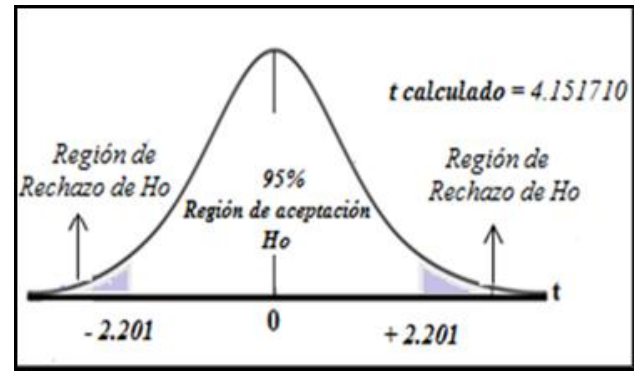

Gráfico 7: La Curva de la comprobación de Hipótesis (Campana de Gauss)

Elaborado por: Autores

Como se puede observar en la campana de Gauss del grafico 7, el valor de, $\mathrm{t}$ calculado $=$ 4.151710; se halla ubicado en la zona de región de rechazo de $\mathrm{H} 0$, superior al valor crítico de la tabla $=2.201$; a un nivel de significancia de 5\%; por cuanto, se rechaza la hipótesis nula a favor de la hipótesis alterna.

Se puede concluir que, "La Inmigración impactó positivamente en el crecimiento de la Renta Per Cápita del Ecuador, en el período 2001-2015; ya que al incrementarse en 1\% el factor demográfico de inmigración, el PIB per cápita aumenta un $0.21 \%$.

\section{Comentarios finales}

La Inmigración presenta un comportamiento ascendente a partir del año 2001 al 2015, de 640.561 inmigrantes a 1.544.463 respectivamente, procedentes principalmente de Cuba, Colombia, China, Haití y otros países americanos ya sea por motivos laborales, académicos o servicios profesionales, que se han visto atraídos por la moneda principalmente. 
El crecimiento de la renta per cápita en el Ecuador muestra una tendencia creciente positiva en los últimos quince años, con una tasa promedio del 4\%, pero en el año 2009 se reduce un punto porcentual, debido a la crisis mundial, para el año 2015 se ubica en 4,298.00 dólares por persona.

El coeficiente parcial del factor demográfico extranjero, evidencia la influencia positiva de la inmigración en el crecimiento de la renta per cápita del Ecuador, la cual, muestra que un incremento de $1 \%$ en el número de inmigrantes generaría un aumento de $0.21 \%$ en el PIB per cápita.

Finalmente es necesario que se estimule la inversión extranjera, otorgando los incentivos tributarios a fin de que los inmigrantes influyan en el sistema productivo, en la implementación de equipos tecnológicos y el conocimiento, generando mayor ocupación y el consumo de bienes y servicios, y por lo tanto un crecimiento de la renta per cápita y el bienestar de la población.

\section{Bibliografía}

Avendaño, L. (2007). Inmigración y Renta Per cápita.

CEPAL. (2014). Comisión Económica para América Latina y el Caribe. Obtenido de http://www.cepal.org/es/datos-y-estadisticas

CEPAL. (2015). Comisión Económica para América Latina y el Caribe. Obtenido de http://www.cepal.org/es/datos-y-estadisticas

Dornbusch. (2008). Macroeconomía. México: McGraw-Hill Companies Inc.

Instituto Nacional de Estadística y Censos. (2010). Obtenido de http://www.inec.gob.ec

Oglietti, G. (2006). Crecimiento, demografía e inmigración: estimación en datos de panel para la Unión Europea.

Oyarzun, J. (2008). Causas y Efectos Económicos de la Inmigración: Un análisis teórico-empírico. Madrid.

Patiño, F. (2014). ¿Realidad o Discurso? Los derechos humanos de los Migrantes Centroamericanos en México. Mexico D.F.

Revista Analítika. (2015). Obtenido de http://www.ecuadorencifras.gob.ec/Analitika/ 